\title{
Rely XTM U200 versus Rely XTM ARC: uma comparação da resistência à microtração
}

\author{
Rely XTM U200 versus Rely XTM ARC: a comparison of microtensile bond \\ strength
}

\author{
Eduardo DA-RÉa (1), Kellen Cristina da Silva GASQUE ${ }^{a}$, Rafael Tobias MORETTI NETOa* (1) \\ aUNIFAL - Universidade Federal de Alfenas, Faculdade de Odontologia, Alfenas, MG, Brasil
}

Como citar: Da-Ré E, Gasque KCS, Moretti Neto RT. Rely XTM U200 versus Rely XTM ARC: uma comparação da resistência à microtração. Rev Odontol UNESP. 2019;48:e20180132. https://dx.doi.org/10.1590/1807-2577.13218

\begin{abstract}
Resumo
Introdução: Os cimentos resinosos são extensivamente utilizados na fixação de restaurações indiretas de cerâmica e cerômero, sendo classificados em duas categorias: os cimentos resinosos convencionais, que não apresentam uma adesão inerente à estrutura dental e requerem o uso de um sistema adesivo e os cimentos resinosos autoadesivos, que não requerem um tratamento adesivo prévio do substrato dentário. Objetivo: Avaliar a resistência de união de dois cimentos resinosos, convencional e autoadesivo, quando utilizados na cimentação de restaurações cerâmicas e ceroméricas, trazendo elementos para propiciar melhor compreensão da interação adesiva em procedimentos de cimentação com as referidas categorias de cimentos resinosos. Material e método: Dentes humanos $(\mathrm{n}=20)$, cedidos pelo banco de dentes da Universidade Federal de Alfenas (UNIFAL), foram preparados para que cilindros de cerâmica ou de cerômero fossem cimentados utilizando-se dois tipos de cimentos resinosos (autoadesivo e convencional). Após a cimentação, 20 palitos foram obtidos e submetidos ao teste de tração, avaliando-se a força necessária para a fratura. Os valores obtidos foram submetidos à análise estatística empregando-se a análise de variância (ANOVA) e o teste de Tukey. Resultado: As forças mínimas e máximas necessárias ao rompimento do cimento convencional foram superiores às mesmas requeridas para o rompimento da união promovida pelo cimento autoadesivo, bem como as medianas e as médias aritméticas, independentemente do material restaurador empregado. 0 teste de Tukey demonstrou que as diferenças entre os cimentos empregados são estatisticamente significantes, independentemente do material restaurador. Conclusão: Os resultados deste trabalho sugerem que o material utilizado para a confecção dos corpos de prova (cerâmica ou cerômero) não influenciou na resistência à tração, sendo que o cimento convencional apresentou valores superiores de resistência.
\end{abstract}

Descritores: Microtração; cimentos resinosos; restaurações indiretas.

\begin{abstract}
Introduction: Resin cements are extensively used in the fixation of indirect restorations of ceramics and ceramics, divided into two categories: conventional resin cements, which do not present an inherent adhesion to the dental structure and require the use of an adhesive system, and the self-adhesive resin cements, which do not require a prior adhesive treatment of the dental substrate. Objective: To evaluate the microtensile bond strength of two resin-based cements, conventional and self-adhesive, according to required operating protocols, when luting ceramic and indirect composite restorations. Material and method: Human molars $(n=20)$ donated by UNIFAL teeth bank were prepared and, after being divided into two groups, ceramic or composite cylinders were luted to them with two resin-based cements (conventional and self-adhesive). Prepared teeth were sectioned vertically in both buccal-lingual and mesio-distal directions, to obtain $8 \mathrm{~mm}$ high, square-shaped "sticks" that were subject to microtensile bond strength test, and the necessary force to fracture them was evaluated. Results were subject to twoway ANOVA and Tukey test. Result: Minimum and maximum forces to disrupt conventional cement were greater than the required one for self-adhesive cement, as well as mean bond strength, despíte of the restorative material. Tukey test showed that diferences were statistically significant, regardless the restorative material. Conclusion: Restorative materials did not influence microtensile bond strength values, with no statistically significant differences. The difference between microtensile bond strength values of resin cements tested was statistically significant.
\end{abstract}

Descriptors: Micro-tensile; bond strength; resin cements. 


\section{INTRODUÇÃO}

Os cimentos resinosos são extensivamente utilizados na fixação de restaurações indiretas de cerâmica e cerômero, devido à adequada interação com os substratos dentários, à baixa solubilidade, à biocompatibilidade, à facilidade de manuseio e à estética ideal, quando comparados aos cimentos tradicionais ${ }^{1,2}$.

O conceito de cimentação em Odontologia refere-se ao uso de uma substância moldável para vedar um espaço ou fixar dois componentes de constituição diferente, evitando-se a penetração de fluidos orais e a invasão bacteriana ${ }^{3}$. Os cimentos utilizados devem apresentar uma combinação de propriedades físicas, químicas e biológicas adequadas: baixa viscosidade, mínima microinfiltração, baixa solubilidade em ambiente aquoso, maior retenção e resistência à fratura ${ }^{1}$.

Os cimentos resinosos são compostos por resinas de Bis-GMA e outros metacrilatos, em combinação com monômeros de baixa viscosidade e cargas inorgânicas (lítio, alumínio e óxido de silício), tratadas com silano ${ }^{3}$. As partículas inorgânicas representam um peso que varia entre 36 a 77\% e têm diâmetro variável, dependendo do produto ${ }^{4}$. Os cimentos autoadesivos possuem natureza mais semelhante aos compômeros, com diferença na concentração dos monômeros ácidos e menor teor de carga, para proporcionar uma menor espessura da linha de cimento, além de serem mais hidratados, facilitando a ionização dos monômeros ácidos, por meio da neutralização da reação ácido-base que envolve o dente e o cimento ${ }^{5}$.

Tais materiais podem ser classificados em duas categorias: os cimentos resinosos convencionais, que não apresentam uma adesão inerente à estrutura dental e requerem o uso de um sistema adesivo, e os cimentos resinosos autoadesivos, que não requerem um tratamento adesivo prévio do substrato dentário ${ }^{6-8}$. Os cimentos resinosos convencionais podem ser utilizados com três tipos de sistema adesivo: a) constituído por agente condicionante, primer e adesivo propriamente dito; b) agente condicionante ácido e um componente que contém primer e adesivo no mesmo frasco, e c) sem condicionamento ácido prévio ${ }^{9-11}$. Os cimentos resinosos autoadesivos dispensam qualquer tratamento dentário prévio ${ }^{12,13}$.

Outro tipo de classificação utilizada para estes cimentos é a sua polimerização: ativados quimicamente, fotoativados ou duais (combinação de ambos os tipos de reação). Os fotoativáveis têm sua polimerização iniciada apenas quando expostos à luz do aparelho fotoativador, com tempo de trabalho controlável $^{3}$, porém limitada profundidade de polimerizaçãoํ. Já os cimentos duais atingem uma profundidade de polimerização maior, com controle do tempo de trabalho ${ }^{1}$.

A técnica de cimentação com cimentos resinosos convencionais, por demandar muitas etapas operatórias, é um procedimento muito susceptível a erros, os quais podem comprometer a resistência de união adequada entre a cerâmica e o substrato dental. Falhas ou intercorrências, em qualquer uma das etapas, podem prejudicar a hibridização da dentina e comprometer o desempenho clínico da restauração ${ }^{14}$.

Uma variedade de cimentos resinosos com desempenho clínico bastante satisfatório já foi desenvolvida4,15,16, exigindo atenção na escolha do melhor material para determinada aplicação clínica. Pensando nisso, este estudo busca avaliar a resistência de união de dois cimentos resinosos, convencional e autoadesivo, de acordo com os protocolos operacionais requeridos para esses cimentos, quando da cimentação de restaurações cerâmicas e ceroméricas, verificando se o tipo de material poderá alterar ou não o desempenho do cimento. Além disso, busca-se trazer elementos adicionais para propiciar melhor compreensão da interação adesiva em procedimentos de cimentação com as referidas categorias de cimentos resinosos. 


\section{MATERIAL E MÉTODO}

Este estudo foi desenvolvido no Laboratório de Pesquisas da Faculdade de Odontologia da Universidade Federal de Alfenas-MG, após a aprovação do Comitê de Ética em Pesquisas

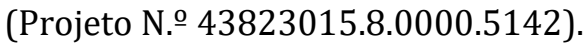

Neste estudo, foram utilizados os cimentos resinosos: convencional Rely $\mathrm{X}^{\circledR}$ ARC e autoadesivo Rely $\mathrm{X}^{\circledR} \mathrm{U} 200$.

Dentes molares permanentes humanos livres de cárie, trincas ou fraturas foram obtidos do Banco de Dentes Humanos da Faculdade de Odontologia da UNIFAL, sendo mantidos sob refrigeração $\left(5^{\circ} \mathrm{C}\right)$ em água destilada até o momento de sua utilização.

A porção oclusal da coroa dos dentes foi removida com um corte no sentido transversal, utilizando-se a fossa central da face oclusal como referência de profundidade, com o uso de um disco de diamante (Isomet Buehler Ltda., Lake Bluf, IL, EUA), em uma cortadeira de precisão (Extec Labcut 150 - Extec/USA). Após o corte, os dentes foram numerados e moldados pela técnica de um passo, utilizando-se material à base de silicona de condensação (Zetaplus/Oranwash - Zhermac, Itália), com um tubo de PVC adaptado para moldagem. Os modelos foram confeccionados com gesso G4 tipo IV especial para troquéis (SS White Artigos Dentários - Rio de Janeiro-RJ, Brasil) e divididos aleatoriamente em dois grupos, com 10 unidades cada um.

Blocos de vidro-cerâmica e leucita EX-3 Press cor A3 (Noritake Kizai CO. - Nagoya, Japão) e blocos de cerômero Ceramage cor A3 (Shofu Dental Corporation - Japão) foram produzidos sobre estes modelos, com verificação da adaptação (Figura 1D). Cada grupo foi novamente dividido aleatoriamente em dois subgrupos de cinco unidades e, em cada dente, foi cimentado um bloco, utilizando-se os cimentos do estudo.
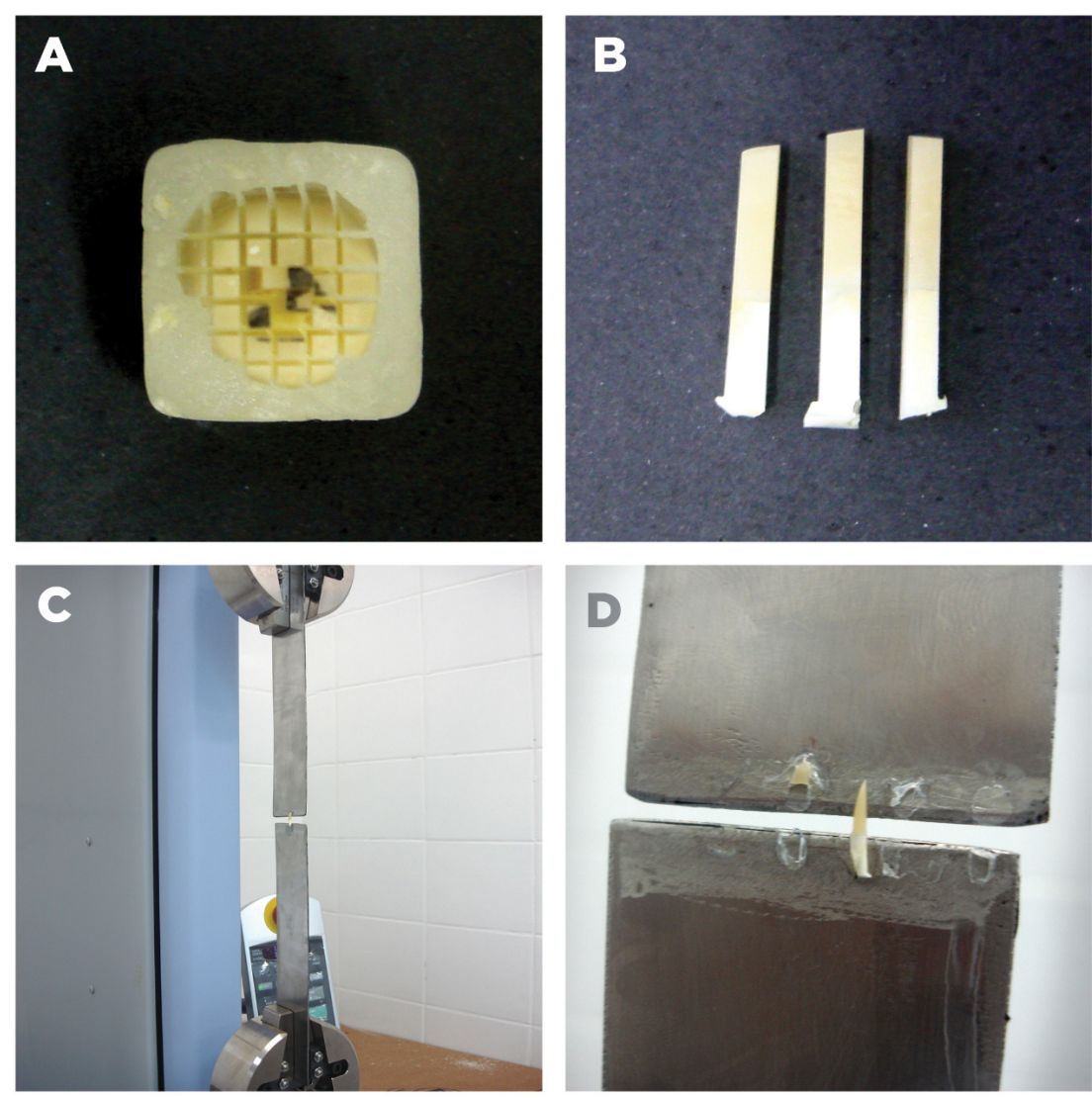

Figura 1. (A) Detalhe do dente com bloco após a realização dos cortes; (B) Palitos obtidos após o corte;

(C) Máquina preparada para o experimento com as hastes de aço inoxidável em posição; (D) Palito posicionado com o auxílio de adesivo Super Bonder Gel. 
Os blocos de cerômero foram condicionados com duas aplicações do agente de união silano por um minuto, intercaladas com secagem por jatos de ar. Os blocos de cerâmica também receberam as aplicações do silano, mas foram previamente tratados com Condicionador de Porcelanas (ácido fluorídrico a $10 \%$, da Dentsply) por quatro minutos, lavados com spray água/ar e secos com jato de ar por 10 segundos.

Os dentes que receberam o cimento convencional foram condicionados com ácido fosfórico a $37 \%$ por 20 segundos, sendo lavados com spray água/ar e secos com ar livre de óleo por cinco segundos. Então, uma primeira aplicação de adesivo foi feita e seca com jato de ar por dois segundos, sendo reaplicada e polimerizada por cinco segundos. Os dentes que receberam o cimento autoadesivo não foram condicionados.

Para padronização da cimentação, foi utilizada a máquina de ensaios EZ Test EZ-LX (Shimadzu Corporation - Japão) na função de compressão, aplicando-se uma força de $10 \mathrm{~N}$ sobre o conjunto bloco-dente após a aplicação do cimento e mantida até a polimerização do mesmo.

Assim, foram obtidos quatro grupos: dois com cerômero, sendo Grupo I (cimentado com Rely X ARC) e Grupo II (cimentado com Rely X U200), e dois com cerâmica, sendo Grupo III (cimentado com Rely X ARC) e Grupo IV (cimentado com Rely X U200).

Após 24 horas de armazenamento em estufa $\left(37^{\circ} \mathrm{C}\right.$ em água destilada), os dentes foram levados à cortadeira metalográfica, em que receberam cortes longitudinais seriados, com o objetivo de padronizar os espécimes, perpendiculares à face oclusal, tanto no sentido mesiodistal como no sentido vestibulolingual, utilizando-se um disco diamantado, sob irrigação constante. Os espécimes resultantes foram removidos da base radicular com um corte transversal, obtendo-se palitos com base quadrada de aproximadamente 1,5 $\mathrm{mm}$ de lado e altura aproximada de 6 a $8 \mathrm{~mm}$ (Figuras 1A e 1B). Os palitos da região periférica dos dentes, ou seja, aqueles que apresentavam união em esmalte, foram descartados do estudo. Os espécimes que apresentavam defeitos na interface ou próximos desta foram excluídos do estudo, após observação em lupa estereoscópica com aumento de 40 vezes.

De cada um dos quatro grupos, selecionaram-se aleatoriamente 20 espécimes em forma de palito, calculando-se a área com um paquímetro digital (Marberg, São Paulo-SP, Brasil) (Figura 1B). Os palitos foram fixados em hastes de aço inoxidável com o auxílio de adesivo à base de cianoacrilato (Superbonder Power Flex gel - Loctite Henkel - São Paulo-SP, Brasil) e tracionados em máquina universal de ensaios à velocidade de $1 \mathrm{~mm} / \mathrm{min}$, até o momento da fratura (Figuras 1C e 1D). Os valores de carga máxima suportada pela união dentina/material restaurador foram calculados em newton $(\mathrm{N})$ pelo software TrapeziumX (Shimadzu Corporation - Japão), dividindo-se a força máxima atingida no momento da fratura pela área da interface adesiva $\left(\mathrm{mm}^{2}\right)$.

Um resumo dos grupos experimentais é apresentado na Figura 2.

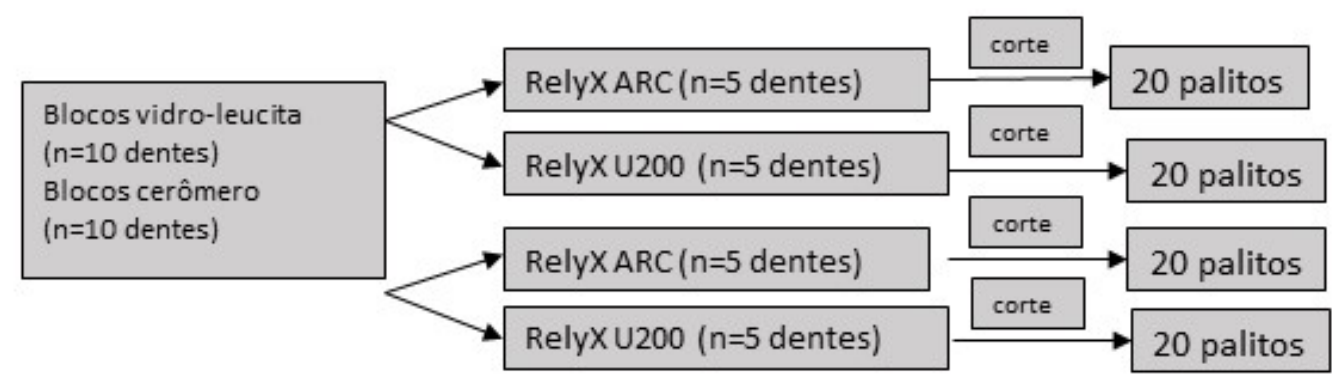

Figura 2. Resumo dos grupos experimentais, desde a seleção dos dentes até a produção dos palitos para os ensaios. 


\section{ANÁLISE DE DADOS}

Os resultados obtidos a partir do teste de tração (resistência à união) foram submetidos à análise estatística, empregando-se o teste paramétrico ANOVA a dois critérios de classificação, no sentido de identificar ou não probabilidades de igualdades ou diferenças entre as condições experimentais. Para comparações individuais entre os diferentes grupos do estudo, os dados foram submetidos ao teste de Tukey. 0 nível de significância para rejeição da hipótese de nulidade foi fixado em $5 \%(\mathrm{p}<0,05)$.

\section{RESULTADO}

Os resultados do teste de microtração (médias e desvio padrão) são apresentados na Tabela 1.0 teste de Tukey mostrou que houve diferença significativa entre a resistência adesiva dos cimentos resinosos na interface adesiva $(p=0,0006)$. Ou seja, houve diferença estatística para as análises entre os grupos I e II, bem como entre os grupos III e IV.

Tabela 1. Resultados da resistência de união à microtração: médias e desvios padrões dos Grupos I, II, III e IV

\begin{tabular}{ccccc}
\hline & Grupo I & Grupo II & Grupo III & Grupo IV \\
\hline Número de Amostras & 20 & 20 & 20 & 20 \\
Desvio padrão & 3.8455 & 1.5142 & 5.0447 & 1.8707 \\
Média & 7.4489 & 3.5056 & 6.5291 & 3.3548 \\
\hline
\end{tabular}

\section{DISCUSSÃO}

Os cimentos resinosos conquistaram uma parcela significativa do mercado odontológico por atenderem a uma exigência estética dos pacientes, por possuírem praticidade de manuseio pelos cirurgiões-dentistas e por sua efetividade clínica ${ }^{5,12,16-18}$. Os cimentos autoadesivos dispensam diversas etapas durante sua aplicação, favorecendo seu desempenho clínico. No entanto, interagem com a dentina proporcionando um menor potencial de adesão ${ }^{18}$, característica que pode ser melhorada significativamente com o condicionamento por ácido fosfórico a 37\% 17 , aumentando a "molhabilidade" da dentina, permitindo uma melhor infiltração do cimento resinoso após a remoção da smear layer na camada condicionada.

Para melhorar a infiltração do cimento, é recomendado o controle da viscosidade ${ }^{17}$. Neste estudo, o grupo do cimento resinoso convencional, aliado à técnica de hibridização, apresentou resistência à tração superior à dos grupos em que se utilizou o cimento resinoso autoadesivo. Tais resultados podem ser explicados pela menor interação cimento/dentina, pela ausência de tags de resina ou de camada híbrida nos cimentos autoadesivos, enquanto que, nos cimentos convencionais, ocorre uma hibridização de dentina superior a $2 \mu \mathrm{m}^{16}$.

Durante a cimentação, foi aplicada uma força de $10 \mathrm{~N}$ (valor compatível com as situações clínicas) ao conjunto dente-cimento-restauração, para aumentar o contato entre as superfícies, promover um maior escoamento do cimento e assim "forçar" uma maior penetração do mesmo nos túbulos dentinários, conforme recomendação de trabalhos anteriores 19,20. Como consequência desses baixos valores de microtração, corroborados em nosso estudo para todos os corpos de prova utilizados, é recomendável uma cuidadosa avaliação clínica e precaução na utilização de cimentos resinosos autoadesivos ${ }^{17}$.

0 cimento convencional apresenta maior resistência à microtração, quando comparado a várias marcas de cimentos autoadesivos ${ }^{21,22}$. Restaurações indiretas produzidas pelo sistema CAD-CAM apresentaram maior resistência à fratura por cisalhamento 23 e à 
microtração ${ }^{24}$, quando foram utilizados os cimentos resinosos convencionais, em comparação aos autoadesivos. Esses dados foram confirmados em nosso trabalho, variandose o condicionamento aplicado à restauração de resina composta que foi cimentada em dentes humanos.

Outro fator que merece consideração é a longevidade clínica dos cimentos autoadesivos. Visto serem estes materiais relativamente novos no mercado, poucos estudos clínicos estão disponíveis. Dados os valores significantemente menores encontrados no presente estudo para o cimento autoadesivo, é possível inferir a possibilidade de complicações pós-operatórias em longo prazo, quando do uso clínico dos cimentos autoadesivos. Outros estudos mostraram resultados superiores de resistência à microtração quando da utilização de cimentos autoadesivos, em comparação ao cimento tradicional de fosfato de zinco ${ }^{24,25}$. Resultados semelhantes foram encontrados em estudo de termoenvelhecimento, em que houve uma tendência à diminuição da resistência à tração dos cimentos resinosos, principalmente os autoadesivos $^{22}$. No presente estudo, não foi avaliado o efeito do termoenvelhecimento dos cimentos resinosos, mas sim sua efetividade após a cimentação. Apesar disso, é possível afirmar que a cimentação adesiva seja fortemente influenciada pelo tipo de material empregado e que a utilização deste material possa determinar a longevidade das restaurações indiretas em situações clínicas.

Em estudo de resistência ao cisalhamento, o tipo de cimento utilizado afetou significativamente a união dos materiais cerâmicos à dentina, resultando em valores superiores para o RelyX U200 (o mesmo utilizado no presente estudo), quando comparados com os valores de Maxcem Elite e com os não resinosos Duo-Link, Panavia F 2.0, e RelyX Ultimate Clicker ${ }^{26}$.

A secagem da dentina também pode influenciar na resistência à microtração, sendo indicado que se evitem tanto o secamento excessivo da dentina quanto a presença de água, quando do uso de cimentos resinosos autoadesivos e convencionais $24,26,27$. No presente estudo, evitou-se a desidratação da dentina, sendo a secagem realizada pelo emprego de jatos de ar durante 10 segundos, conforme recomendado pelos fabricantes, permitindo a interação dos materiais com o substrato dentinário.

\section{CONCLUSÃO}

O material utilizado para a confecção dos corpos de prova não influenciou nos valores de resistência à tração, sendo que o cimento resinoso convencional Rely XTM ARC apresentou valores superiores de resistência à tração tanto para cerâmica quanto para cerômero.

\section{AGRADECIMENTOS}

O presente trabalho foi realizado com recursos próprios.

\section{REFERÊNCIAS}

1. Haddad MF, Rocha EP, Assunção WG. Cementation of prosthetic restorations: from conventional cementation to dental bonding concept. J Craniofac Surg. 2011 May;22(3):952-8. http://dx.doi.org/10.1097/SCS.0b013e31820fe205. PMid:21558917.

2. Melo RM, Özcan M, Barbosa SH, Galhano G, Amaral R, Bottino MA, et al. Bond strength of two resin cements on dentin using different cementation strategies. J Esthet Restor Dent. 2010 Aug;22(4):262-8. http://dx.doi.org/10.1111/j.1708-8240.2010.00349.x. PMid:20690956.

3. Anusavice KJ. Phillips, materiais dentários. 11aㅡ ed. Rio de Janeiro: Elsevier; 2005. 
4. Van Meerbeek B, Inokoshi S, Davidson CL, De Gee AJ, Lambrechts P, Braem M, et al. Dual cure luting composites - part II: clinically related properties. J Oral Rehabil. 1994 Jan;21(1):57-66. http://dx.doi.org/10.1111/j.1365-2842.1994.tb01124.x. PMid:8133389.

5. Ferracane JL, Stansbury JW, Burke FJ. Self-adhesive resin cements - chemistry, properties and clinical considerations. J Oral Rehabil. 2011 Apr;38(4):295-314. http://dx.doi.org/10.1111/j.13652842.2010.02148.x. PMid:21133983.

6. Manso AP, Silva NR, Bonfante EA, Pegoraro TA, Dias RA, Carvalho RM. Cements and adhesives for allceramic restorations. Dent Clin North Am. 2011 Apr;55(2):311-32, ix. http://dx.doi.org/10.1016/j.cden.2011.01.011. PMid: 21473996.

7. Perdigao J, Geraldeli S, Hodges JS. Total-etch versus self-etch adhesive: effect on postoperative sensitivity. J Am Dent Assoc. 2003 Dec;134(12):1621-9. http://dx.doi.org/10.14219/jada.archive.2003.0109. PMid:14719760.

8. Van Meerbeek B, Yoshihara K, Yoshida Y, Mine A, De Munck J, Van Landuyt KL. State of the art of selfetch adhesives. Dent Mater. 2011 Jan;27(1):17-28.; published online Nov 24, 2010. http://dx.doi.org/10.1016/j.dental.2010.10.023. PMid:21109301.

9. Silva e Souza MH Jr, Carneiro KG, Lobato MF, Silva e Souza PA, de Góes MF. Adhesive systems: important aspects related to their composition and clinical use. J Appl Oral Sci. 2010 MayJun;18(3):207-14. http://dx.doi.org/10.1590/S1678-77572010000300002. PMid:20856995.

10. Pashley DH, Tay FR, Breschi L, Tjäderhane L, Carvalho RM, Carrilho M, et al. State of the art of etch-andrinse adhesives. Dent Mater. 2011 Jan;27(1):1-16. http://dx.doi.org/10.1016/j.dental.2010.10.016. PMid:21112620.

11. Peumans M, Kanumilli P, De Munck J, Van Landuyt K, Lambrechts P, Van Meerbeek B. Clinical efectiveness of contemporary adhesives: a systematic review of current clinical trials. Dent Mater. 2005 Sep;21(9):864-81. http://dx.doi.org/10.1016/j.dental.2005.02.003. PMid:16009415.

12. Radovic I, Monticelli F, Goracci C, Vulicevic ZR, Ferrari M. Self-adhesive resin cements: a literature review. J Adhes Dent. 2008 Aug;10(4):251-8. PMid:18792695.

13. Gerth HU, Dammaschke T, Züchner H, Schäfer E. Chemical analysis and bonding reaction of RelyX Unicem and Bifix composites - a comparative study. Dent Mater. 2006 Oct;22(10):934-41. http://dx.doi.org/10.1016/j.dental.2005.10.004. PMid:16364427.

14. Anchieta RB, Rocha EP, de Almeida EO, Freitas AC Jr, Martini AP. Bonding all-ceramic restorations with two resins cement techniques: a clinical report of three-year follow- up. Eur J Dent. 2011 Aug;5(4):478-85. PMid:21912505.

15. Pameijer CH, Stanley HR. Pulp reactions to resin cements. Am J Dent. 1992 Apr;5(2):81-7. PMid:1524752.

16. Aguiar TR, Andre CB, Arrais CAG, Bedran-Russo AK, Giannini M. Micromorphology of resin-dentin interfaces using self-adhesive and conventional resin cements: A confocal laser and scanning electron microscope analysis. Int J Adhes Adhes. 2012 Oct;38:69-74.

http://dx.doi.org/10.1016/j.ijadhadh.2012.05.009.

17. Pisani-Proença J, Erhardt MC, Amaral R, Valandro LF, Bottino MA, Del Castillo-Salmerón R. Influence of different surface conditioning protocols on microtensile bond strength of self-adhesive resin cements to dentin. J Prosthet Dent. 2011 Apr;105(4):227-35. http://dx.doi.org/10.1016/S00223913(11)60037-1. PMid:21458647.

18. Burgess JO, Ghuman T, Cakir D, Swift EJ Jr. Self-adhesive resin cements. J Esthet Restor Dent. 2010 Dec;22(6):412-9. http://dx.doi.org/10.1111/j.1708-8240.2010.00378.x. PMid:21171499.

19. De Munck J, Vargas M, Van Landuyt K, Hikita K, Lambrechts P, Van Meerbeek B. Bonding of an autoadhesive luting material to enamel and dentin. Dent Mater. 2004 Dec;20(10):963-71. http://dx.doi.org/10.1016/j.dental.2004.03.002. PMid:15501325. 
20. Goracci C, Cury AH, Cantoro A, Papacchini F, Tay FR, Ferrari M. Microtensile bond strength and interfacial properties of self-etching and self-adhesive resin cements used to lute composite onlays under different seating forces. J Adhes Dent. 2006 Oct;8(5):327-35. PMid:17080881.

21. Fuentes MV, Ceballos L, González-López S. Bond strength of self-adhesive resin cements to different treated indirect composites. Clin Oral Investig. 2013 Apr;17(3):717-24. http://dx.doi.org/10.1007/s00784-012-0752-y. PMid:22619093.

22. Qeblawi DM, Campillo-Funollet M, Muñoz CA. In vitro shear bond strength of two self-adhesive resin cements to zirconia. J Prosthet Dent. 2015 Feb;113(2):122-7. http://dx.doi.org/10.1016/j.prosdent.2014.08.006. PMid:25438742.

23. Skupien JA, Porto JA, Münchow EA, Cenci MS, Pereira-Cenci T. Impairment of resin cement application on the bond strength of indirect composite restorations. Braz Oral Res. 2015;29(1):1-7. http://dx.doi.org/10.1590/1807-3107BOR-2015.vol29.0066. PMid:26039908.

24. Kim JY, Cho GY, Roh BD, Shin Y. Effect of curing mode on shear bond strength of self-adhesive cement to composite blocks. Materials (Basel). 2016 Mar;9(3):E210. http://dx.doi.org/10.3390/ma9030210. PMid:28773334.

25. Gundogdu M, Aladag LI. Effect of adhesive resin cements on bond strength of ceramic core materials to dentin. Niger J Clin Pract. 2018 Mar;21(3):367-74. http://dx.doi.org/10.4103/njcp.njcp_10_17. PMid:29519988.

26. Hitz T, Stawarczyk B, Fischer J, Hämmerle CH, Sailer I. Are self-adhesive resin cements a valid alternative to conventional resin cements? A laboratory study of the long-term bond strength. Dent Mater. 2012 Nov;28(11):1183-90. http://dx.doi.org/10.1016/j.dental.2012.09.006. PMid:22999370.

27. Ayyildiz S, Emir F, Tunc EP, Sen D. Shear bond strength of various luting cements to fixed prosthodontic restorative materials. Appl Adhes Sci. 2015;3(1):13. http://dx.doi.org/10.1186/s40563015-0039-z

\section{CONFLITOS DE INTERESSE}

Os autores declaram não haver conflitos de interesse.

\section{*AUTOR PARA CORRESPONDÊNCIA}

Rafael Tobias Moretti Neto, UNIFAL - Universidade Federal de Alfenas, Faculdade de Odontologia, Rua Gabriel Monteiro da Silva, 700, 37130-001 Alfenas - MG, Brasil, e-mail: rafmoreti@unifal-mg.edu.br

Recebido: Dezembro 5, 2018

Aprovado: Janeiro 17, 2019 